\title{
Theocharis Problem Reconsidered in Differentiated Oligopoly
}

\author{
Akio Matsumoto ${ }^{1}$ and Ferenc Szidarovszky ${ }^{2}$ \\ ${ }^{1}$ Department of Economics, Institute of Further Development of Dynamic Economic Research, Chuo University, 742-1 Higashi-Nakano, \\ Tokyo 192-0393, Japan \\ ${ }^{2}$ Department of Applied Mathematics, Universty of Pécs, Ifjúság u-6, Pécs 7624, Hungary
}

Correspondence should be addressed to Akio Matsumoto; akiom@tamacc.chuo-u.ac.jp

Received 2 April 2014; Accepted 20 July 2014; Published 17 August 2014

Academic Editor: Bernard Fortin

Copyright (C) 2014 A. Matsumoto and F. Szidarovszky. This is an open access article distributed under the Creative Commons Attribution License, which permits unrestricted use, distribution, and reproduction in any medium, provided the original work is properly cited.

\begin{abstract}
Oligopolies with product differentiation are examined with both quantity-adjusting and price-adjusting firms. Conditions are given for the positivity of the equilibrium outputs as well as for that of the equilibrium prices. The stability of the positive equilibria is then investigated; the positivity and stability conditions are also compared. The dependence of these conditions on the number of firms, their qualifications and the degree of relation between the goods is discussed. The analysis is given by assuming both discrete- and continuous-time scales.
\end{abstract}

\section{Introduction}

In his seminal paper, Theocharis [1] constructs the $n$-firm Cournot oligopoly model with a linear demand function and constant marginal costs and studies the asymptotic stability of the steady state under discrete-time quantity adjustment. It is then found that stability depends only on the number of firms; the steady state is globally stable in the duopoly, marginally stable in the triopoly, and destabilized if the number of firms becomes more than three. It is also pointed out that this provocative result (sometimes called the Theocharis problem) might be obtained under the limited environment in which the firms have full and instantaneous information on the demand function, their own cost, and the competitors' costs, the goods are perfectly substitutes (i.e., homogenous), and expectations are naively formed. As a natural consequence, the Theocharis model has been extended in various directions to weaken these restrictions. Focusing on the type of the adjustment process, McManus and Quandt [2] and Hahn [3] prove that the steady state is asymptotically stable in the continuous-time adjustment process with demand and cost functions having the appropriate slopes. It is also confirmed in Fisher [4] that the steady state can be stabilized when the adjustment coefficient is small and/or the marginal cost is increasing in the discrete-time adjustment process. Further, models with product differentiation are discussed in Hadar [5], Krelle [6], Okuguchi [7], Sridhar Moorthy [8], and Friedman [9] among others. Models with product differentiations can be considered special cases of multiproduct oligopolies, which were first formulated by Selten [10] for quantity strategies and by Eichhorn [11, 12] for price strategies. Szidarovszky et al. [13] examine the stability of equilibria in linear multiproduct oligopolies with adaptive adjustments and derive sufficient and necessary stability conditions. A comprehensive summary of the main results on the existence, uniqueness, and stability of equilibria in multiproduct oligopolies is given in Okuguchi and Szidarovszky [14] which can be easily applied to oligopolies with product differentiation as special cases. Goldberg [15] presents an application to the US Automobile Industry. Comparison of Bertrand and Cournot oligopolies is discussed in Okuguchi [16], Vives [17], and Cheng [18] among others. More recently, taking into account of the nonnegativity constraints on the variables, Cánovas et al. [19] consider the global dynamics to show that the $n$-firm model has simple dynamics (i.e., it converges either to a monopoly or a duopoly or to a two-periodic oscillation), whose results are further extended in Cánovas [20].

Our purpose of this study is to reconsider the Theocharis problem in differentiated oligopoly, following the spirit of 
Hadar [5] in which the steady state is shown to be stable if the degree of differentiation is weak and the coefficient matrix of the naive dynamic system has a dominant diagonal under the general forms of the demand and cost functions. In this study, we take away the assumption of the diagonal dominance but restore the linear structure of the Theocharis model where demand is linear and marginal costs are constant. Introducing product differentiation with microfoundation leads to an environment in which the firms are also differentiated; each firm faces its own demand function and they operate with different production costs. These two sorts of differentiation affect dynamics. Further we introduce the price adjustment (i.e., Bertrand competition) in addition to the quantity adjustment (i.e., Cournot competition) to investigate the effects caused by the choice of operational strategies and then address dynamics in the continuous-time scales. In short, we raise a question whether the Theocharis problem still exists under those broader circumstances.

The rest of the paper is organized as follows. Section 2 briefly gives a microfoundation of the linear demand functions of the differentiated goods. Section 3 presents an $n$-firm linear oligopoly model in Cournot competition and examines asymptotic dynamics in the extended model. Section 4 considers the Theocharis problem in Bertrand competitions. Concluding remarks are given in Section 5.

\section{Consumers}

It is assumed that there is a continuum of consumers of the same type and the utility function of the representative consumer is given as

$$
U(\mathbf{q}, I)=\sum_{i=1}^{n} \alpha_{i} q_{i}-\frac{1}{2}\left(\sum_{i=1}^{n} q_{i}^{2}+2 \gamma \sum_{i=1}^{n} \sum_{j>i}^{n} q_{i} q_{j}\right)-I,
$$

where $\mathbf{q}=\left(q_{i}\right)$ is the quantity vector, $I=\sum_{i=1}^{n} p_{i} q_{i}$ with $p_{i}$ being the price of good $i, \alpha_{i}$ measures the quality of good $i$, and $\gamma \in[-1,1]$ measures the degree of relation between the goods: $\gamma>0, \gamma<0$, or $\gamma=0$ imply that the goods are substitutes, complements, or independent. Moreover, the goods are perfect substitutes if $\gamma=1$ and perfect complements if $\gamma=-1$. In this study, we confine our analysis to the case in which the goods are imperfect substitutes or complements and are not independent, by assuming that $|\gamma|<1$ and $\gamma \neq 0$.

The linear inverse demand function (or the price function) of good $k$ is obtained from the first-order condition of the interior optimal consumption of good $k$ and is given by

$$
p_{k}=\alpha_{k}-q_{k}-\gamma \sum_{i \neq k}^{n} q_{i} \text { for } k=1,2, \ldots, n
$$

where $n \geq 2$ is assumed. More compactly, (2) can be written in the vector form; the price vector is a linear function of the output vector:

$$
\mathbf{p}=\alpha-\mathbf{B q}
$$

where $\mathbf{p}=\left(p_{i}\right), \boldsymbol{\alpha}=\left(\alpha_{i}\right)$, and $\mathbf{B}=\left(B_{i j}\right)$ with $B_{i i}=1$ and $B_{i j}=\gamma$ for $i \neq j$. Since $\mathbf{B}$ is invertible ${ }^{1}$, solving (3) for $\mathbf{q}$ yields the direct demand

$$
\mathbf{q}=\mathbf{B}^{-1}(\boldsymbol{\alpha}-\mathbf{p}),
$$

where the diagonal and the off-diagonal elements of $\mathbf{B}^{-1}$ are, respectively,

$$
\frac{1+(n-2) \gamma}{(1-\gamma)(1+(n-1) \gamma)}, \quad-\frac{\gamma}{(1-\gamma)(1+(n-1) \gamma)}
$$

Hence the direct demand of good $k$, the $k$ th-component of $\mathbf{q}$, is linear in the prices and is given as

$$
\begin{array}{r}
q_{k}=\frac{(1+(n-2) \gamma)\left(\alpha_{k}-p_{k}\right)-\gamma \sum_{i \neq k}^{n}\left(\alpha_{i}-p_{i}\right)}{(1-\gamma)(1+(n-1) \gamma)} \\
\text { for } k=1,2, \ldots, n .
\end{array}
$$

For the sake of the later analysis, let us define the admissible region of $(\gamma, n)$ by $D_{(+)}$or $D_{(-)}$according to whether the goods are substitutes or complements:

$$
\begin{gathered}
D_{(+)}=\{(\gamma, n) \mid 0<\gamma<1,2 \leq n\}, \\
D_{(-)}=\{(\gamma, n) \mid-1<\gamma<0,2 \leq n\} .
\end{gathered}
$$

\section{Quantity-Adjusting Firms}

In Cournot competition, firm $k$ chooses a quantity $q_{k}$ to maximize its profit $\pi_{k}=\left(p_{k}-c_{k}\right) q_{k}$ subject to its price function (2), taking the other firms' quantities given. We assume a linear cost function for each firm, so that the marginal cost $c_{k}$ is constant and nonnegative. To avoid negative optimal production, we also assume that $\alpha_{k}-c_{k}$ is positive.

Assumption 1 ( $c_{k} \geq 0$ and $\alpha_{k}-c_{k}>0$ for all $k$ ). Assuming interior maximum and solving its first-order condition yield the best reply of firm $k$,

$$
q_{k}=\frac{\alpha_{k}-c_{k}}{2}-\frac{\gamma}{2} \sum_{i \neq k}^{n} q_{i} \text { for } k=1,2, \ldots, n .
$$

It can be easily checked that the second-order condition is certainly satisfied. The Cournot equilibrium output and price for firm $k$ are obtained by solving the following simultaneous equations:

$$
q_{k}+\frac{\gamma}{2} \sum_{i \neq k}^{n} q_{i}=\frac{\alpha_{k}-c_{k}}{2} \text { for } k=1,2, \ldots, n,
$$

or in vector form

$$
\mathbf{B}^{C} \mathbf{q}=\mathbf{A}^{C},
$$

where $\mathbf{A}^{C}=\left(\left(\alpha_{i}-c_{i}\right) / 2\right)$ and $\mathbf{B}^{C}=\left(B_{i j}^{C}\right)$ with $B_{i i}^{C}=1$ and $B_{i j}^{C}=\gamma / 2$ for $i \neq j . \quad \mathbf{B}^{C}$ is invertible if $2+(n-1) \gamma \neq 0$ and, in this case, the Cournot output vector is given by

$$
\mathbf{q}^{C}=\left(\mathbf{B}^{C}\right)^{-1} \mathbf{A}^{C},
$$


where the diagonal and off-diagonal elements of $\left(\mathbf{B}^{C}\right)^{-1}$ are, respectively,

$$
\frac{2(2+(n-2) \gamma)}{(2-\gamma)(2+(n-1) \gamma)}, \quad-\frac{2 \gamma}{(2-\gamma)(2+(n-1) \gamma)} .
$$

Hence the Cournot equilibrium output of firm $k$ is

$$
q_{k}^{C}=\frac{\alpha_{k}-c_{k}}{2-\gamma}-\frac{\gamma}{(2-\gamma)(2+(n-1) \gamma)} \sum_{i=1}^{n}\left(\alpha_{i}-c_{i}\right)
$$

and the Cournot equilibrium price of firm $k$ is

$$
p_{k}^{C}=\frac{\alpha_{k}+c_{k}-\gamma c_{k}}{2-\gamma}-\frac{\gamma}{(2-\gamma)(2+(n-1) \gamma)} \sum_{i=1}^{n}\left(\alpha_{i}-c_{i}\right) .
$$

Subtracting (13) from (14) yields $p_{k}^{C}-c_{k}=q_{k}^{C}$ and then, by substituting it into the profit function, the Cournot profit becomes

$$
\pi_{k}^{C}=\left(q_{k}^{C}\right)^{2}
$$

3.1. Nonnegativity. Before proceeding to the stability analysis, we check the nonnegativity conditions for the variables at the Cournot equilibrium point. The relations $p_{k}^{C}-c_{k}=q_{k}^{C}$ and (15) imply that the Cournot price and the Cournot profit are positive if the Cournot output is positive. So it suffices to show $q_{k}^{C} \geq 0$. We start with the case of $\gamma>0$ in which $2+(n-1) \gamma>0$ always. Equation (13) can be written as

$$
q_{k}^{C}=\frac{\alpha_{k}-c_{k}}{(2-\gamma)(2+(n-1) \gamma)}\left\{2-\gamma+\left(1-\beta_{k}\right) n \gamma\right\},
$$

where $\beta_{k}$ is defined as

$$
\beta_{k}=\frac{(1 / n) \sum_{i=1}^{n}\left(\alpha_{i}-c_{i}\right)}{\alpha_{k}-c_{k}} .
$$

The constant term $\alpha_{k}$ of the price function is the maximum price of the product $q_{k}$ and thought to be a proxy of the product quality (i.e., the high-price product is usually highly qualified) and thus $\alpha_{k}-c_{k}$ could measure the net quality. $\beta_{k}$ is the ratio of the average net quality over the individual net quality of firm $k$. When $\beta_{k}<1$, firm $k$ is called higherqualified as its individual net quality is larger than the average net quality. On the other hand, when $\beta_{k}>1$, firm $k$ is called lower-qualified as the individual net quality is less than the average net quality. Equation (16) implies that $q_{k}^{C}>0$ if $\beta_{k} \leq 1$. A different form of (16) is

$$
q_{k}^{C}=\frac{\left(\alpha_{k}-c_{k}\right)\left(\beta_{k}-1\right) \gamma}{(2-\gamma)(2+(n-1) \gamma)}\left\{\frac{2-\gamma}{\gamma\left(\beta_{k}-1\right)}-n\right\}
$$

and leads to $q_{k}^{C}>0$ if $\beta_{k}>1$ and

$$
n<\frac{2-\gamma}{\gamma\left(\beta_{k}-1\right)}
$$

We call the locus of $(\gamma, n)$ satisfying relation (19) with equality the zero-output curve.

We now turn to the case of $\gamma<0$ in which the sign of $2+(n-1) \gamma$ is ambiguous. Equation (13) indicates $q_{k}^{C}>0$ if $2+(n-1) \gamma>0$. Positivity of the output does not depend on whether the firm is higher- or lower-qualified if

$$
n<1-\frac{2}{\gamma} .
$$

We now suppose the opposite situation where $2+(n-1) \gamma<$ 0 . Then (16) and $\beta_{k} \geq 1$ together imply $q_{k}^{C}<0$. The lowerqualified firms temporarily choose zero-production and will exit the market if nothing is changed. Decreasing the number of firms could reverse the inequality of the condition: $2+(n-$ 1) $\gamma<0$, and, in consequence, the remaining firms produce positive output. On the other hand, (18) implies that $q_{k}^{C}>0$ if $\beta_{k}<1$ and

$$
n>\frac{2-\gamma}{\gamma\left(\beta_{k}-1\right)} .
$$

Solving $2+(n-1) \gamma=0$ for $n$ and subtracting the resultant expression from the right hand side of (21) present

$$
\frac{2-\gamma}{\gamma\left(\beta_{k}-1\right)}-\left(1-\frac{2}{\gamma}\right)=\frac{(2-\gamma) \beta_{k}}{(-\gamma)\left(1-\beta_{k}\right)}>0 .
$$

This inequality implies that the zero-output curve with $\gamma<0$ is in the region of $2+(n-1) \gamma<0$. Hence the nonnegativity conditions for Cournot output are summarized as follows.

Lemma 2. In the case of $\gamma>0$, Cournot output of a firm is positive if

$$
\text { either the firm is higher-qualified (i.e., } \beta_{k}<1 \text { ) }
$$

or

it is lower-qualified (i.e., $\beta_{k}>1$ ) and $n<\frac{2-\gamma}{\gamma\left(\beta_{k}-1\right)}$,

while, in the case of $\gamma<0$, it is positive if

$$
\text { either } n<1-\frac{2}{\gamma}
$$

or

$$
\text { the firm is lower-qualified (i.e., } \beta_{k}<1 \text { ) and } n>\frac{2-\gamma}{\gamma\left(\beta_{k}-1\right)} \text {. }
$$

3.2. Stability. We now turn attention to the stability of the Cournot output. Best response dynamics is assumed with naive expectations, when each firm believes that the other firms remain unchanged with their outputs from the previous period. By assuming discrete-time scales, the best response (8) gives rise to the time invariant linear dynamic system

$$
q_{k}(t+1)=\frac{\alpha_{k}-c_{k}}{2}-\frac{\gamma}{2} \sum_{i \neq k}^{n} q_{i}(t), \quad k=1,2, \ldots, n .
$$




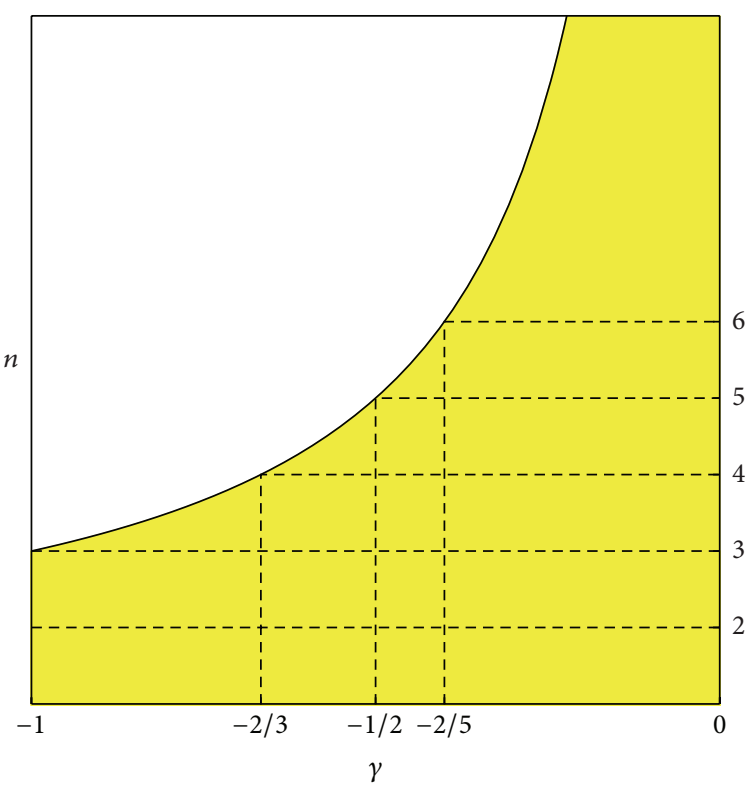

(a) Complements: $\gamma<0$

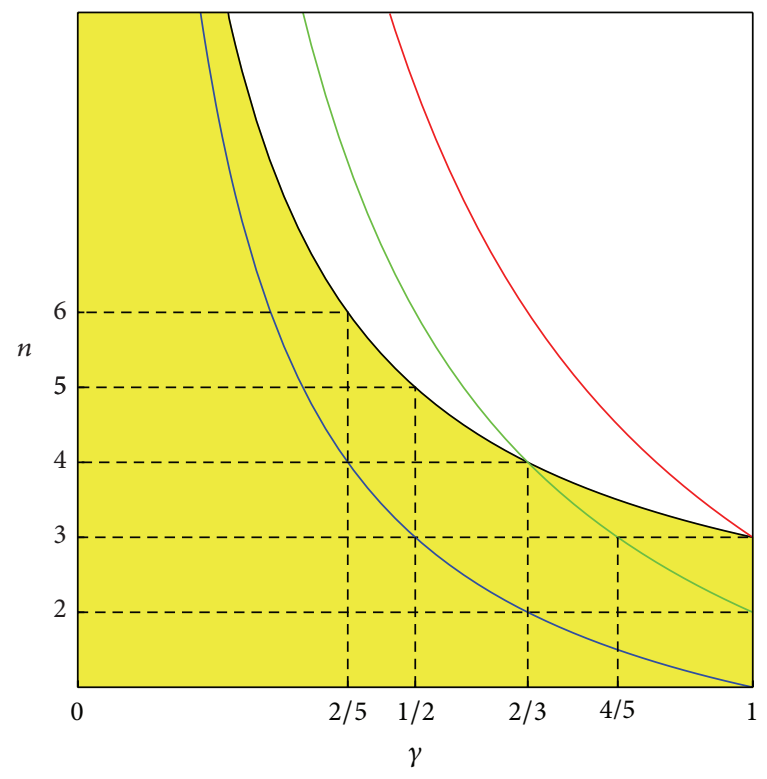

(b) Substitutes: $\gamma>0$

FIGURE 1: Cournot competition: stability and nonnegativity region.

Substituting $q_{k}(t+1)$ into the price function (2) of firm $k$ yields the price dynamic equation associated with the output dynamics:

$$
\begin{array}{r}
p_{k}(t+1)=\alpha_{k}-q_{k}(t+1)-\gamma \sum_{i \neq k}^{n} q_{i}(t+1), \\
k=1,2, \ldots, n .
\end{array}
$$

Equations (27) and (28) imply that the price dynamics is essentially the same as the quantity dynamics. In other words, the Cournot price is stable (resp., unstable) if the Cournot output is stable (resp., unstable). Therefore it is enough for our purpose to draw attention only to the stability of the Cournot output.

The coefficient matrix of system (27) is

$$
\mathbf{J}_{C}=\left(\begin{array}{cccc}
0 & -\frac{\gamma}{2} & \cdots & -\frac{\gamma}{2} \\
-\frac{\gamma}{2} & 0 & \cdots & -\frac{\gamma}{2} \\
\cdot & \cdot & \cdots & \cdot \\
-\frac{\gamma}{2} & -\frac{\gamma}{2} & \cdots & 0
\end{array}\right) .
$$

The corresponding characteristic equation reads as follows:

$$
\left|\mathbf{J}_{C}-\lambda \mathbf{I}\right|=(-1)^{n}\left(\lambda-\frac{\gamma}{2}\right)^{n-1}\left(\lambda+\frac{(n-1) \gamma}{2}\right)=0,
$$

which indicates that there are $n-1$ identical eigenvalues and one different eigenvalue. Without loss of generality, the first $n-1$ eigenvalues are assumed to be identical:

$$
\lambda_{1}^{C}=\lambda_{2}^{C}=\cdots=\lambda_{n-1}^{C}=\frac{\gamma}{2}, \quad \lambda_{n}^{C}=-\frac{(n-1) \gamma}{2} .
$$

Since $|\gamma|<1$ is assumed, the first $n-1$ eigenvalues are less than unity in absolute value. It depends on the absolute value of $\lambda_{n}^{C}$ whether the Cournot output is stable or not. It is clear that $\left|\lambda_{n}^{C}\right|<1$ for $n=2$ and 3. Solving $\left|\lambda_{n}^{C}\right|<1$ for $n>3$ presents the stability conditions of the Cournot output ${ }^{2}$ :

$$
n<1+\frac{2}{\gamma} \quad \text { if } \gamma>0, \quad n<1-\frac{2}{\gamma} \quad \text { if } \gamma<0 .
$$

We can now summarize these stability results as follows.

Lemma 3. In Cournot competition with discrete-time scales, the Cournot output is stable, marginally stable, or unstable according to whether the number of the firms is less than, equal to, or greater than $1+2 /|\gamma|$.

Lemma 2 is concerned with the nonnegativity condition for the Cournot output and so is Lemma 3 with the stability condition. Combining these lemmas provides the condition for which the Cournot output is positive and stable. We now investigate the following effects on stability of the positive Cournot output: the differentiation effect caused by a change in $\gamma$, the heterogeneity effect caused by a change in $\beta_{k}$, and the time scale effect caused by changing the time scale to continuous from discrete.

3.3. Differentiation Effect. To see the differentiation effect on stability, we assume away the heterogeneity of the firms for a while only for analytical simplicity by assuming that $\alpha_{k}=\alpha$ and $c_{k}=c$ for all $k$. This simplification leads to $\beta_{k}=1$ for all $k$. Due to (16), $q_{k}^{C}>0$ for all $k$ if $\gamma>0$ or if $\gamma<0$ and $2+(n-1) \gamma>0$. The positive-sloping black curve in Figure 1(a) and the negative-sloping black curve in 
Figure $1(\mathrm{~b})$ are the corresponding $\left|\lambda_{n}^{C}\right|=1$ or marginalstability curves. The yellow regions under the black curves are the stability regions. Thus, the positive Cournot output is always stable if $n=2$ or $n=3$ and the goods are neither perfect substitutes $(\gamma=1)$ nor perfect complements $(\gamma=-1)$. The second result implies that the Theocharis problem does not arise when the goods are differentiated (i.e., $|\gamma| \neq 1$ ) in the triopoly market in which (27) exhibits marginal stability when $|\gamma|=1$. For $n \geq 4$, stability depends on the degree of the differentiation. Solving $\left|\lambda_{n}^{C}\right|=1$ gives the threshold value of $\gamma$ :

$$
\left|\gamma_{n}^{C}\right|=\frac{2}{n-1}
$$

For example, when $n$ increases to 6 from 4 with an increment of unity, the threshold value decreases to $2 / 3,1 / 2$, and $2 / 5$, and Cournot output is stable if

$$
\begin{gathered}
|\gamma|<\frac{2}{3} \quad \text { when } n=4, \quad|\gamma|<\frac{1}{2} \quad \text { when } n=5, \\
|\gamma|<\frac{2}{5} \quad \text { when } n=6
\end{gathered}
$$

and becomes unstable when the inequality is reversed. In short, Cournot output for $n \geq 4$ can be stable if the goods are strongly differentiated in the sense that the degree of differentiation in absolute value is less than $2 /(n-1)$. A larger value of $n$ could be a destabilizer in the sense that the stability region shrinks as $n$ increases. Nevertheless, the differentiation partially settles the Theocharis problem. Further, observing Figures 1(a) and 1(b), we see that the differentiation effect is symmetric; that is, one figure is the mirror image of the other.

Lemma 4. Supposing that $\beta_{k}=1$ for all $k$, the Cournot output is positive and stable if the degree of differentiation satisfies

$$
|\gamma|<\frac{2}{n-1}
$$

3.4. Heterogeneity Effect. Since $\beta_{k}=1$ for all $k$ is a special case, we examine the heterogeneity effect of $\beta_{k} \neq 1$ on stability. For $\gamma>0$, Cournot output is always positive if $\beta_{k}<1$. The stability condition of the positive Cournot output produced by the higher-qualified firm is the same as the one with $\beta_{k}=1$ :

$$
n<1+\frac{2}{\gamma}
$$

Graphically the Cournot output is positive and stable in the yellow region of Figure 1(b) and unstable in the white region. The heterogeneity among the higher-qualified firms does not affect stability.

When firm $k$ is lower-qualified (i.e., $\beta_{k}>1$ ), the stability region is accordingly modified. The zero-output curve and the marginal-stability curve yield

$$
\frac{2-\gamma}{\gamma\left(\beta_{k}-1\right)}-\left(1+\frac{2}{\gamma}\right)=\frac{2+\gamma}{\gamma\left(\beta_{k}-1\right)}\left(\frac{4}{2+\gamma}-\beta_{k}\right),
$$

where the first factor on the right hand side is positive for $\beta_{k}>$ 1 and the second factor is negative if $\beta_{k}>2$ and positive if $4 / 3<\beta_{k}<2$. We distinguish three cases, Case I where $\beta_{k}>2$, Case II where $\beta_{k}<4 / 3$, and Case III where $4 / 3<$ $\beta_{k}<2$. In Case I, the nonnegativity condition is stronger than the stability condition. Stability of the Cournot output can be examined with the consideration of the nonnegativity condition. The negative-sloping blue curve in Figure 1(b) is the zero-output curve with $\beta_{k}=2$. The Cournot output is positive and stable in the yellow region under the zerooutput curve. It is still stable in the yellow region between the zero-output curve and the marginal-stability curve but it is negative there. Notice that, as $\beta_{k}$ becomes larger than 2 , the blue curve shifts downward making the nonnegativity region smaller. The heterogeneity $\beta_{k}>2$ can be a destabilizer.

Lemma 5. Supposing that $\beta_{k}>2$, the Cournot output is positive and stable if

$$
n<\frac{2-\gamma}{\gamma\left(\beta_{k}-1\right)} \text {. }
$$

Next we draw attention to Case II. The zero-output curve with $\beta_{k}=4 / 3$ is in red and located above the marginalstability (black) curve in Figure 1(b). It can be verified that the zero-output curve shifts upward as $\beta_{k}$ decreases. Thus, for $1<$ $\beta_{k}<4 / 3$, (37) implies that the stability condition is stronger than the nonnegativity condition. In Figure 1(b), the Cournot output is positive and stable in the yellow region below the black curve. The stability conditions of the higher-qualified firm (i.e., $\beta_{k}<1$ ) and the lower-qualified firms (i.e., $\beta_{k}>1$ ) are the same. However the optimal behavior is different when the stability condition is violated. The Cournot output of the lower-qualified firm is negative in the white region above the red curve so that the firm chooses to produce nothing and may exit the market. Further, it is positive and unstable in the white region between the red curve and the black curve. As is seen above, the higher-qualified firm produces positive output which is unstable, implying that the firm may stay in the market even if the stability condition is violated. Notice that changing $\beta_{k}$ does not affect the stability condition and thus has no heterogeneity effect.

Lemma 6. Supposing that $1<\beta_{k}<4 / 3$, the Cournot output is positive and stable if

$$
n<1+\frac{2}{\gamma}
$$

In Case III, the zero-output curve intersects the marginalstability curve at the threshold value $\gamma_{k}$ determined as

$$
\gamma_{k}=\frac{2\left(2-\beta_{k}\right)}{\beta_{k}}
$$

where $\gamma_{k} \rightarrow 1$ as $\beta_{k} \rightarrow 4 / 3$ and $\gamma_{k} \rightarrow 0$ as $\beta_{k} \rightarrow 2$. For $\gamma>\gamma_{k}$, the Cournot output satisfying $n<1+2 / \gamma$ is positive and stable and, for $\gamma<\gamma_{k}$, the Cournot output satisfying $n<(2-\gamma) /\left[\gamma\left(\beta_{k}-1\right)\right]$ is positive and stable. In Figure 1(b), the negative-sloping green curve is the zero-output curve with 
$\beta_{k}=3 / 2$, intersects the black curve at the point of $\gamma_{k}=2 / 3$ and $n=4$, and passes through the point of $\gamma=1$ and $n=2$. The alternative expression of the zero-output curve is

$$
\gamma_{n}^{0}=\frac{2}{1+\left(\beta_{k}-1\right) n}
$$

where, given $\beta_{k}=3 / 2$,

$$
\begin{gathered}
\gamma_{2}^{0}=1 \quad \text { for } n=2, \quad \gamma_{3}^{0}=\frac{4}{5} \quad \text { for } n=3, \\
\gamma_{4}^{0}=\frac{2}{3} \quad \text { for } n=4 .
\end{gathered}
$$

Thereby the Cournot output is always positive and stable for any $\gamma \in(0,1)$ in the duopoly market (i.e., $n=2)$. It is positive and stable for $\gamma<\gamma_{3}^{0}$ in the triopoly market (i.e., $n=3$ ). For $\gamma>\gamma_{3}^{0}$ it is stable but negative. So firm $k$ chooses to produce nothing. For $n=4$, notice that $\gamma_{4}=\gamma_{4}^{0}$. Cournot output is positive and stable for $\gamma<\gamma_{4}^{0}$ and chosen to be zero for $\gamma>\gamma_{4}^{0}$, leading to the firm's exit from the market. For $n=5$, Cournot output is positive and stable for $\gamma<\gamma_{5}$, positive and unstable for $\gamma_{5}<\gamma<\gamma_{5}^{0}$, and negative and unstable for $\gamma>\gamma_{5}^{0}$. We then have the hybrid result that the Cournot output is positive and stable for $\gamma<\min \left(\gamma_{n}, \gamma_{n}^{0}\right)$ and is zero for $\gamma>\gamma_{k}^{0}$.

Lemma 7. Supposing that $4 / 3<\beta_{k}<2$, the Cournot output is positive and stable if

$$
n<\min \left\{1+\frac{2}{\gamma}, \frac{2-\gamma}{\gamma\left(\beta_{k}-1\right)}\right\} \text {. }
$$

In the case of $\gamma<0$, the situation is much simpler since the nonnegativity condition is identical with the stability condition. In Figure 1(a), the positive sloping black curve is not only the zero-output curve but also the marginal-stability curve. The positive Cournot output is stable in the yellow region below the black curve. The heterogeneity effect among the firms (i.e., $\beta_{k} \neq 1$ ) provides a sharp contrast in whether the goods are substitutes or complements. In summary, the heterogeneity affects stability of positive Cournot output by shifting the zero-output curve when $\gamma>0$ and has no effect on stability when $\gamma<0$. Therefore we can say that heterogeneity partially settles the Theocharis problem only when the goods are substitutes. Lemmas 2-7 lead to the main result obtained under the Cournot competition.

Theorem 8. In the case of $\gamma>0$, the Cournot output is positive and stable one of the following exclusive conditions holds:

(i) $\beta_{k}<4 / 3, \quad n<1+\frac{2}{\gamma}$,

(ii) $\frac{4}{3}<\beta_{k}<2, \quad n<\min \left\{1+\frac{2}{\gamma}, \frac{2-\gamma}{\gamma\left(\beta_{k}-1\right)}\right\}$,

(iii) $\beta_{k}>2, \quad n<\frac{2-\gamma}{\gamma\left(\beta_{k}-1\right)}$, while, in the case of $\gamma<0$, it is stable, irrespective of the value of $\beta_{k}$,

$$
\text { if } n<1-\frac{2}{\gamma} \text {. }
$$

3.5. Time Scale Effect. If continuous-time scales are assumed by replacing $q(t+1)-q(t)$ by $\dot{q}(t)$, then system (27) is modified as follows:

$$
\dot{q}_{k}(t)=\frac{\alpha_{k}-c_{k}}{2}-\frac{\gamma}{2} \sum_{i \neq k} q_{i}(t)-q_{k}(t) .
$$

Notice that the steady state of the continuous system is the same as that of the discrete system. Its Jacobian matrix is

$$
\mathbf{J}_{C}-\mathbf{I}=\left(\begin{array}{cccc}
-1 & -\frac{\gamma}{2} & \cdots & -\frac{\gamma}{2} \\
-\frac{\gamma}{2} & -1 & \cdots & -\frac{\gamma}{2} \\
\cdot & \cdot & \cdots & \cdot \\
-\frac{\gamma}{2} & -\frac{\gamma}{2} & \cdots & -1
\end{array}\right) .
$$

The corresponding characteristic equation has the form

$$
\left|\mathbf{J}_{C}-\mathbf{I}-\lambda \mathbf{I}\right|=(-1)^{n}\left(\lambda+1-\frac{\gamma}{2}\right)^{n-1}\left(\lambda+1+\frac{(n-1) \gamma}{2}\right)=0
$$

implying that the eigenvalues are

$$
-1+\frac{\gamma}{2}, \quad-1-\frac{(n-1) \gamma}{2} .
$$

If $\gamma>0$, then both are negative and, if $\gamma<0$, then they are negative if

$$
n<1-\frac{2}{\gamma}
$$

which is the same stability condition as in the discrete case. We then summarize these results as follows.

Theorem 9. In Cournot competition with continuous-time scales, the Cournot output is stable for any $n \geq 2$ if the goods are substitutes and it is stable for $n<1-2 / \gamma$ if the goods are complements.

Comparing Theorem 8 with Theorem 9 reveals that the time scale effect solves the Theocharis problem since a change of the time scales completely stabilizes the Cournot point in the $D_{(+)}$plane. On the other hand the scale change does not change the stability condition at all if $\gamma<0$. The time scale effect exhibits a sharp difference, depending on whether the goods are substitutes or complements.

\section{Price-Adjusting Firms}

In Bertrand competition, firm $k$ chooses the price of good $k$ to maximize the profit $\pi_{k}=\left(p_{k}-c_{k}\right) q_{k}$ subject to its direct 
demand (6), taking the other firms' prices given. Solving the first-order condition yields the best reply of firm $k$ :

$$
\begin{array}{r}
p_{k}=\frac{\alpha_{k}+c_{k}}{2}-\frac{\gamma}{2[1+(n-2) \gamma]} \sum_{i \neq k}^{n}\left(\alpha_{i}-p_{i}\right), \\
\text { for } k=1,2, \ldots, n .
\end{array}
$$

The second-order condition for an interior optimum solution is

$$
\frac{\partial^{2} \pi_{k}}{\partial p_{k}^{2}}=-\frac{2(1+(n-2) \gamma)}{(1-\gamma)(1+(n-1) \gamma)}<0,
$$

where the direction of inequality depends on the parameter configuration. ${ }^{3}$ For $(\gamma, n) \in D_{(+)}$, we see that (52) is always satisfied. On the other hand, for $(\gamma, n) \in D_{(-)}$, we need an additional condition to fulfill the second-order condition. Since

$$
1+(n-1) \gamma<1+(n-2) \gamma
$$

for $\gamma<0$, the required condition is either $0<1+(n-1) \gamma$ or $1+(n-2) \gamma<0$. Since the first inequality is the stability condition as well as the nonnegativity condition under the Cournot competition, we take it even under the Bertrand competition.

Assumption $10(1+(n-1) \gamma>0$ for $\gamma<0)$. The Bertrand equilibrium prices are obtained by solving the simultaneous equations

$$
\begin{aligned}
p_{k} & -\frac{\gamma}{2[1+(n-2) \gamma]} \sum_{i \neq k}^{n} p_{i} \\
& =\frac{\alpha_{k}+c_{k}}{2}-\frac{\gamma}{2[1+(n-2) \gamma]} \sum_{i \neq k}^{n} \alpha_{i}
\end{aligned}
$$

for $k=1,2, \ldots, n$ with unknowns $p_{k}$. In vector form,

$$
\mathbf{B}^{B} \mathbf{p}=\mathbf{A}^{B},
$$

with $\mathbf{A}^{B}=\left(\left(\alpha_{k}+c_{k}\right) / 2-(\gamma /(2[1+(n-2) \gamma])) \sum_{i \neq k}^{n} \alpha_{i}\right)$ and $\mathbf{B}^{B}=\left(B_{i j}^{B}\right)$, where $B_{i i}^{B}=1$ and $B_{i j}^{B}=-\gamma /(2[1+(n-2) \gamma])$ for $i \neq j$. Since $\mathbf{B}^{B}$ is invertible, the solution is

$$
\mathbf{p}=\left(\mathbf{B}^{B}\right)^{-1} \mathbf{A}^{B}
$$

where the diagonal and off-diagonal elements of $\left(\mathbf{B}^{B}\right)^{-1}$ are, respectively,

$$
\begin{aligned}
& \frac{2(1+(n-2) \gamma)(2+(n-2) \gamma)}{(2+(n-3) \gamma)(2+(2 n-3) \gamma)} \\
& \frac{2 \gamma(1+(n-2) \gamma)}{(2+(n-3) \gamma)(2+(2 n-3) \gamma)}
\end{aligned}
$$

Hence, the Bertrand equilibrium price and output of firm $k$ are given by

$$
\begin{aligned}
p_{k}^{B}= & \left((2+(n-3) \gamma)\left[(1+(n-1) \gamma)\left(\alpha_{k}+c_{k}\right)-\gamma c_{k}\right]\right. \\
& \left.-\gamma(1+(n-2) \gamma) \sum_{i=1}^{n}\left(\alpha_{i}-c_{i}\right)\right) \\
\times & ((2+(2 n-3) \gamma)(2+(n-3) \gamma))^{-1} \\
& q_{k}^{B}=\frac{1+(n-2) \gamma}{(1-\gamma)(1+(n-1) \gamma)}\left(p_{k}^{B}-c_{k}\right)
\end{aligned}
$$

with

$$
\begin{aligned}
p_{k}^{B}-c_{k}= & \left((2+(n-3) \gamma)(1+(n-1) \gamma)\left(\alpha_{k}-c_{k}\right)\right. \\
& \left.-\gamma(1+(n-2) \gamma) \sum_{i=1}^{n}\left(\alpha_{i}-c_{i}\right)\right) \\
& \times((2+(2 n-3) \gamma)(2+(n-3) \gamma))^{-1} .
\end{aligned}
$$

Due to (59), the Bertrand profit of firm $k$ becomes

$$
\pi_{k}^{B}=\frac{(1-\gamma)(1+(n-1) \gamma)}{1+(n-2) \gamma}\left(q_{k}^{B}\right)^{2} .
$$

4.1. Nonnegativity and Stability. Equation (59) implies that the Bertrand output is positive if $p_{k}^{B}-c_{k}$ is positive. $q_{k}^{B} \geq 0$ leads to $p_{k}^{B}>0$ but not vice versa. So we confine our attention to the conditions for $q_{k}^{B} \geq 0$. Equation (60) implies that $p_{k}^{B}-c_{k}$ is always positive if $\gamma<0$ and Assumption 10 holds or it is nonnegative if $\gamma>0$ and

$$
z^{B}(\gamma, n) \geq \beta_{k}
$$

where $\beta_{k}$ is defined by (17) and

$$
z^{B}(\gamma, n)=\frac{(2+(n-3) \gamma)(1+(n-1) \gamma)}{(1+(n-2) \gamma) n \gamma} .
$$

Since it can be confirmed that $z^{B}(\gamma, n)>1$ for $0<\gamma<1$ and $2 \leq n<\infty,{ }^{4}$ the Bertrand price is positive if firm $k$ is higher-qualified or if the firms are homogeneous. For $\beta_{k}>1$, it is positive if $n \leq n_{+}$, where

$$
\begin{aligned}
n_{+}= & \left(3-4 \gamma+\beta_{k}(2 \gamma-1)\right. \\
& \left.+\sqrt{(1-2 \gamma)^{2}\left(\beta_{k}^{2}+1\right)+2 \beta_{k}\left(1-2 \gamma^{2}\right)}\right) \\
& \times\left(2\left(\beta_{k}-1\right)\right)^{-1}
\end{aligned}
$$

is a positive solution of $z^{B}(\gamma, n)=\beta_{k}$. In short, we have the following. 
Lemma 11. In the case of $\gamma>0$, the Bertrand price is positive if

(either the firm is higher-qualified or the firms are all homogenous)

or

$$
\text { the firm is lower-qualified and } n \leq n_{+} \text {, }
$$

while in the case of $\gamma<0$, the Bertrand price is always positive under Assumption 10.

In examining stability of the Bertrand price with discretetime scales, we assume best response dynamics with naive expectations on price formation and obtain the following system of time-invariant difference equations:

$$
\begin{array}{r}
p_{k}(t+1)=\frac{\alpha_{k}+c_{k}}{2}-\frac{\gamma}{2[1+(n-2) \gamma]} \sum_{i \neq k}^{n}\left[\alpha_{i}-p_{i}(t)\right] \\
\text { for } k=1,2, \ldots, n .
\end{array}
$$

Similarly to the Cournot competition, we can also obtain the output difference equations under Bertrand competition by substituting $p_{k}(t+1)$ into the direct demand function (6):

$$
\begin{aligned}
q_{k}(t+1)= & (1+(n-2) \gamma)\left(\alpha_{k}-p_{k}(t+1)\right) \\
& \left.-\gamma \sum_{i \neq k}^{n}\left(\alpha_{i}-p_{i}(t+1)\right)\right) \\
& \times((1-\gamma)(1+(n-1) \gamma))^{-1} .
\end{aligned}
$$

It is clear from (65) and (66) that the output dynamics is synchronized with the price dynamics. We focus on price dynamics henceforth. The coefficient matrix of the priceadjusting system (65) is

$$
\begin{aligned}
& \mathbf{J}_{B}= \\
& \left(\begin{array}{cccc}
0 & \frac{\gamma}{2[1+(n-2) \gamma]} & \cdots & \frac{\gamma}{2[1+(n-2) \gamma]} \\
\frac{\gamma}{2[1+(n-2) \gamma]} & 0 & \cdots & \frac{\gamma}{2[1+(n-2) \gamma]} \\
\cdot & \cdot & \cdots & \cdot \\
\frac{\gamma}{2[1+(n-2) \gamma]} & \frac{\gamma}{2[1+(n-2) \gamma]} & \cdots & 0
\end{array}\right) .
\end{aligned}
$$

This matrix has the same structure as $\mathbf{J}_{C}$. So replacing $\gamma$ of $\lambda_{k}^{C}$ by $-\gamma / 2[1+(n-2) \gamma]$ yields the eigenvalues of $\mathbf{J}_{B}$ :

$$
\begin{gathered}
\lambda_{1}^{B}=\lambda_{2}^{B}=\cdots=\lambda_{n-1}^{B}=-\frac{\gamma}{2[1+(n-2) \gamma]}, \\
\lambda_{n}^{B}=\frac{(n-1) \gamma}{2[1+(n-2) \gamma]} .
\end{gathered}
$$

When $\gamma>0$ and $n \geq 2$, we have $\left|\lambda_{k}^{B}\right|<1$ for $k=1,2, \ldots, n$. That is, the Bertrand price is globally asymptotically stable in $D_{(+)}{ }^{5}$ Neither product differentiation nor homogeneity affects the stability of the Bertrand price if the goods are substitute. When $\gamma<0$ and Assumption 10 is given, $0<\lambda_{k}^{B}<$ 1 holds for $k=1,2, \ldots, n-1$. The value of $\lambda_{n}^{B}$ is clearly negative and the stability condition $\lambda_{n}^{B}>-1$ can be rewritten as

$$
n<\frac{5}{3}-\frac{2}{3 \gamma}
$$

Under Assumption 10, the Bertrand price is stable if the following condition holds: ${ }^{6}$

$$
n<\min \left\{\frac{5}{3}-\frac{2}{3 \gamma}, 1-\frac{1}{\gamma}\right\} .
$$

The stability of the Bertrand price is summarized as follows.

Lemma 12. In Bertrand competition with discrete-time scales, (i) the Bertrand price of a firm is stable for any $n \geq 2$ if the goods are substitutes whereas it is stable if the goods are complements, Assumption 10 holds, and

$$
n<\min \left\{\frac{5}{3}-\frac{2}{3 \gamma}, 1-\frac{1}{\gamma}\right\} .
$$

Lemma 11 is concerned with the nonnegativity condition of the Bertrand price and Lemma 12 is concerned with the stability condition. Thus combining these lemmas gives the following result.

Theorem 13. In the case of $\gamma>0$, the Bertrand price of a firm is positive and stable if

$$
\text { either the firm is higher-qualified or homogenous }
$$

or

$$
\text { the firm is lower-qualified and } n \leq n_{+} \text {, }
$$

while, in the case of $\gamma<0$, it is positive and stable if Assumption 10 holds and

$$
n<\min \left\{\frac{5}{3}-\frac{2}{3 \gamma}, 1-\frac{1}{\gamma}\right\} .
$$

We graphically confirm Theorem 13 . In Figure $2(a)$ in which $\gamma<0$, the positive-sloping red and blue curves correspond, respectively, to the marginal-stability curve and the SOC (i.e., the second-order condition) curve under which Assumption 10 holds. Thus the Bertrand price is positive and stable in the yellow region. It is checked that the two curves intersect for $\gamma=-1 / 2$ and $n=3$. Thus the Bertrand price is always stable in the duopoly and stable if $\gamma>-1 / 2$ in the triopoly. For $n \geq 4$, it is stable if $\gamma>\gamma_{n}^{B}$, where the threshold value $\gamma_{n}^{B}$ is determined by

$$
\gamma_{n}^{B}=\frac{2}{5-3 n}
$$

It is also checked that the Bertrand price is unstable but satisfies Assumption 10 if $1 /(1-n)<\gamma<\gamma_{n}^{B}$ while it is unstable and violates Assumption 10 if $\gamma<1 /(1-n)$. 
In the case of $\gamma>0$, the Bertrand price is stable but its positivity is sensitive to the value of $\beta_{k}$. Indeed, it is positive and stable if the firm is higher-qualified or the firms are all homogenous. On the other hand, if the firm is lowerqualified, then zero-output curve on which $n=n_{+}$divides the $D_{+}$plane, and the Bertrand price is positive and stable only in the region below the curve. In Figure 2(b), three curves with $\beta_{k}=3 / 2, \beta_{k}=5 / 2$, and $\beta_{k}=7 / 2$ are illustrated as three negative-sloping curves. Increasing the value of $\beta_{k}$ shifts the zero-output curve leftward. Thus these colored regions give the stability region of the positive Bertrand price when $\beta_{k}=3 / 2$. The union of the blue and dark-orange regions is the stability region when $\beta_{k}=5 / 2$ and increasing $\beta_{k}$ to $7 / 2$ takes away the blue region, limiting the stability region to the dark-orange region. The stronger heterogeneity makes the stability region smaller through shifting the zerooutput curve leftward. In the $n$-firm model, the Bertrand price is positive and stable for $\gamma<\gamma_{\beta_{k}}$, where $\gamma_{\beta_{k}}$ solves $z^{B}(\gamma, n)=$ $\beta_{k}$. As seen in Figure 2(b), the threshold values of $\gamma$ for $n=2$ are

$$
\begin{gathered}
\gamma_{2 / 3}<-1+\sqrt{3} \simeq 0.732, \quad \gamma_{5 / 2}<-2+\sqrt{6} \simeq 0.449, \\
\gamma_{7 / 2}<-3+\sqrt{11} \simeq 0.317 .
\end{gathered}
$$

It is also seen that larger number of firms is a destabilizing factor as $\gamma_{\beta_{k}}$ is decreasing in $n$.

If continuous-time scales are considered, then the best response dynamics has the Jacobian $\mathbf{J}_{B}-\mathbf{I}$ with eigenvalues

$$
-1-\frac{\gamma}{2(1+(n-2) \gamma)}, \quad-1+\frac{(n-1) \gamma}{2(1+(n-2) \gamma)} .
$$

If $\gamma>0$, then these eigenvalues are negative. If $\gamma<0$, then they are negative if

$$
n<\frac{3}{2}-\frac{1}{\gamma} .
$$

However this inequality always holds under Assumption 10 and thus is ineffective. In summary, a change of the time scales does not affect the stability conditions regardless of whether the goods are substitutes or complements.

Theorem 14. Under Bertrand competition with continuoustime scales, the Bertrand point is stable for any $n$ if the goods are substitutes and for $(\gamma, n)$ satisfying relation $(70)$ if the goods are complements and Assumption 10 holds.

4.2. Strategy Effect. We can consider the strategy effect by comparing Figure 1 with Figure 2. We start with the case of $\gamma<0$. It is easily verified that

$$
\max \left\{\frac{5}{3}-\frac{2}{3 \gamma}, 1-\frac{1}{\gamma}\right\}<1-\frac{2}{\gamma} .
$$

This inequality implies that the marginal stability curve under the Cournot competition is located above the partition curve under the Bertrand competition. In other words, the Cournot output is more stable than the Bertrand price in the sense that the stability region for the positive Cournot output is larger than the stability region for the positive Bertrand price.

On the other hand, in the case of $\gamma>0$, the crucial factor is whether the firm is higher- or lower-qualified. For the higher-qualified firm, the Bertrand price is always positive and stable while the Cournot output is positive and stable if $n<1+2 / \gamma$. Changing the strategy to price adjustment from quantity adjustment works for stabilization. When the firm is lower-qualified, its zero-output curve is obtained from inequality (19) as

$$
n=f\left(\gamma, \beta_{k}\right):=\frac{2-\gamma}{\gamma\left(\beta_{k}-1\right)}
$$

under the Cournot competition and $n=n_{+}$under the Bertrand competition. We abbreviate the former to the $C$ curve and the latter to the $B$-curve. Then solving $f\left(\gamma, \beta_{k}\right)=$ $n_{+}$for $\gamma$ presents the following; the $C$-curve is above and, respectively, below is the $B$-curve according to

$$
\beta_{k}<\frac{2}{\gamma} \text {, resp., } \beta_{k}>\frac{2}{\gamma} \text {. }
$$

The case $2>\beta_{k}(>1)$ is divided into two subcases according to the relative location of the $C$-curve with respect to the marginal-stability curve, $1<\beta_{k}<4 / 3$, and $4 / 3<\beta_{k}<$ 2. The first subcase is depicted by Figure 3(a) in which $\beta_{k}=$ 1.3 is taken and the second case by Figure 3(b) with $\beta_{k}=3 / 2$. In both figures, the red curve is described by $n=f\left(\gamma, \beta_{k}\right)$, the green curve by $n=n_{+}$, and the black curve by the marginalstability condition, $n=1+2 / \gamma$. As shown in Figure 2(b), the $C$-curve is above the black curve implying that the Cournot output is stable in the union of the yellow and blue regions. The $B$-curve crosses the marginal-stability curve from below, adding the red region to the yellow region and subtracting the blue region from it to construct the stability region of the Bertrand price. In the second subcase, the $C$-curve as well as the $B$-curve shifts down by increasing the value of $\beta_{k}$. As a result, as already shown in Figure 2(b), the stability region of the Cournot output is the union of the yellow, blue, and meshed-yellow regions. The stability region of the Bertrand price is the union of the yellow and red regions. Since it may not be determined which is larger, the red region and the blue region, the strategy effect caused by changing the quantity adjustment to the price adjustment on stability is ambiguous when $\beta_{k}<2$.

For $\beta_{k} \geq 2$, the solution of the simultaneous equations $n=f\left(\gamma, \beta_{k}\right)$ and $n=n_{+}$is $\gamma=2 / \beta_{k}$ and $n=2$. As far as $n \geq 2$ is assumed, the $C$-curve is always located above the $B$ curve, implying that the Cournot output is more stable than the Bertrand price in the sense that the stability region under the Cournot competition is larger than the stability region under the Bertrand competition. Summarizing the results, we have the following.

Theorem 15. In the discrete time framework, when the goods are substitutes, the Bertrand competition is more stable for $\beta_{k}<$ 1 , the Cournot competition is more stable for $\beta_{k}>2$, and it is ambiguous which is stronger for $1<\beta_{k}<2$. 


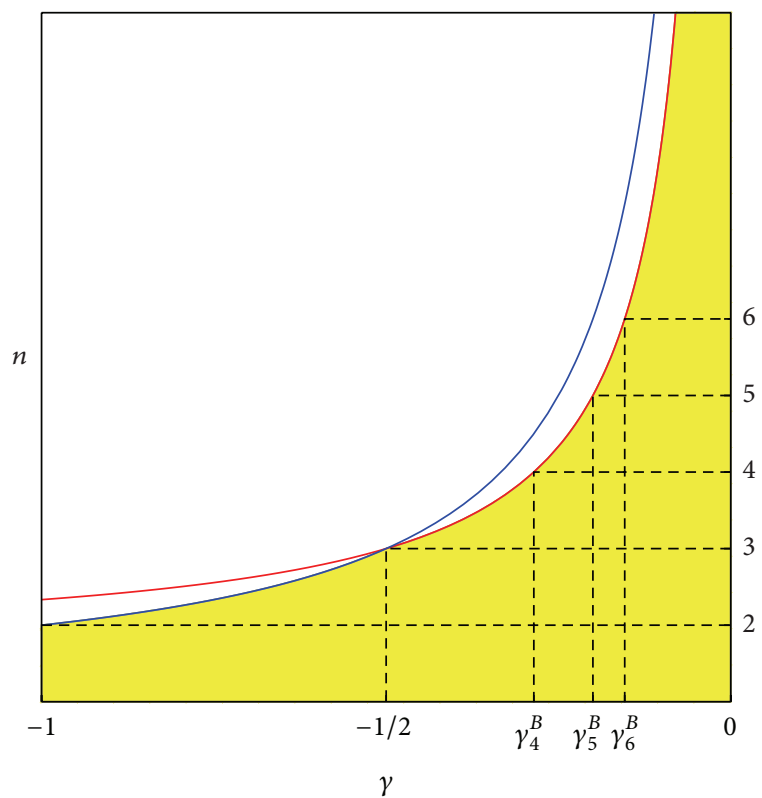

(a) Complements: $\gamma<0$

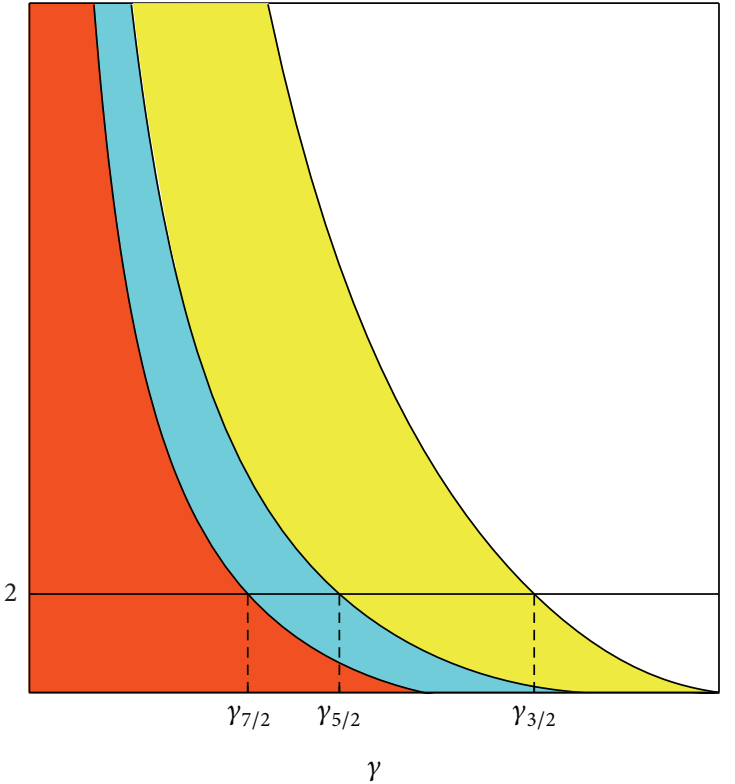

(b) Substitutes: $\gamma>0$

FIGURE 2: Bertrand competition: stability and nonnegativity region.

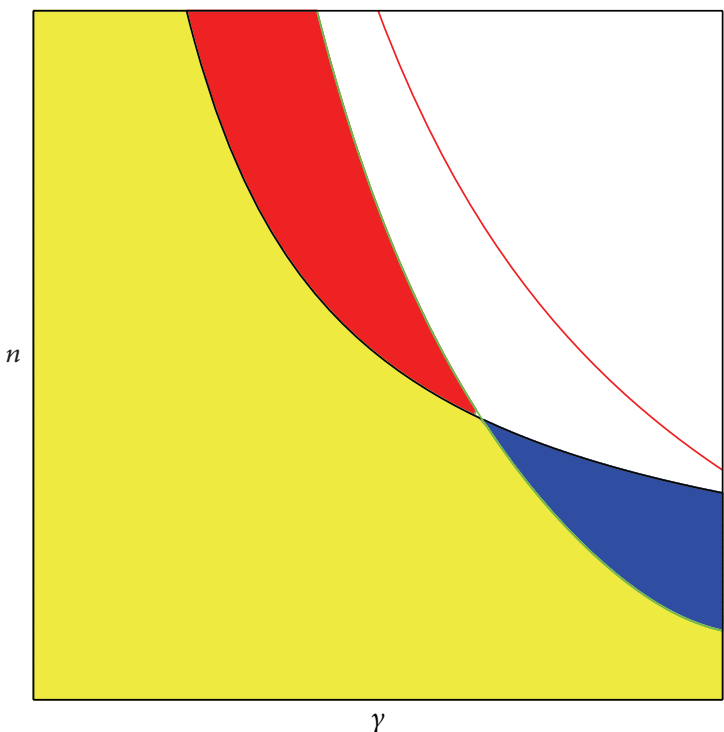

(a) $1<\beta_{k}<4 / 3$

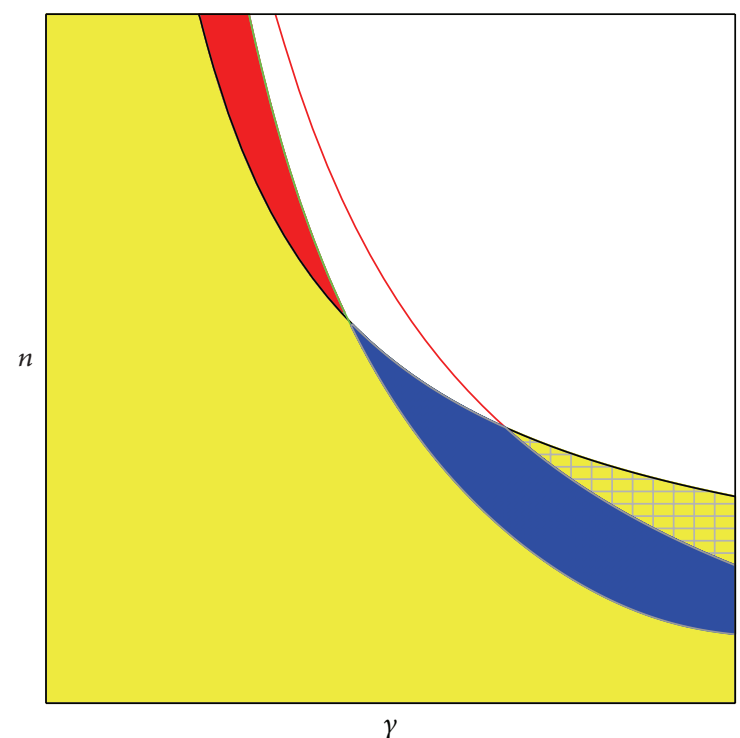

(b) $4 / 3<\beta_{k}<2$

FIGURE 3: Stability regions under Cournot and Bertrand competition.

The Cournot output and Bertrand price are always stable in the continuous-time scale if the goods are substitutes. On the other hand, if the goods are complements, the changing of time scales does not affect the stability conditions in both Cournot and Bertrand competitions. So we have the following.

Theorem 16. In the continuous time framework, both of the Cournot and Bertrand competitions are stable if the goods are substitutes and Cournot competition is more stable than Bertrand competition if the goods are complements.

\section{Concluding Remarks}

Discrete and continuous linear oligopolies are examined with differentiated products. Conditions are derived for the positivity of the equilibrium quantities as well as for that of the equilibrium prices. Then conditions are given for the stability of the positive equilibria. These conditions are analyzed in detail when their dependence on the number of firms $(n)$, on the degree of differentiation of the products $(\gamma)$, on the heterogeneity of the firms $\left(\beta_{k}\right)$, and on the selection of the time scales is examined. In the case of Cournot 
competition our findings can be summarized as follows. With discrete-time scales the positive equilibrium output of a firm is stable if the number of firms is less than a threshold that depends on both $\gamma$ and $\beta_{k}$. If the time scale is continuous, then the positive equilibrium is always stable if the products are substitutes and it is stable if the products are complements and the number of firms is below a certain threshold. In the case of price-adjusting firms and discrete-time scales, we have the following facts. If the products are substitutes, then the positive equilibrium price is stable if the firm is higher qualified $\left(\beta_{k}<1\right)$. If the products are complements, then the positive equilibrium price is stable if the number of firms is below a given threshold. With continuous-time scales the positive equilibrium price is stable for any number of firms if the products are substitutes and with a bounded number of firms if the products are complements. In comparing the stability conditions we can conclude what follows. In the discrete time, framework when the goods are substitutes, the Bertrand competition is more stable for $\beta_{k}<1$ and the Cournot competition is more stable for $\beta_{k}>2$; however, for $1<\beta_{k}<2$, no clear comparison is possible. In the continuous time framework both the Cournot and Bertrand equilibria are stable if the goods are substitutes and Cournot competition is more stable than Bertrand competition if the goods are complements.

Since all models resulted in linear dynamics, stability of the equilibria means global asymptotical stability. Our next research project will be to analyze nonlinear oligopolies and investigate their local and global stability. Further the nonnegativity of the outputs of the firms as well as taking capacity limits into account can be done similarly to Cánovas [20] and Bischi et al. [21]. The resulting model becomes nonlinear making asymptotic analysis a bit more complicated, which will be worked out in our future research.

\section{Conflict of Interests}

The authors declare that there is no conflict of interests regarding the publication of this paper.

\section{Acknowledgments}

The authors thank the referee for the useful comments. They highly appreciate the financial supports from the MEXTSupported Program for the Strategic Research Foundation at Private Universities 2013-2017, the Japan Society for the Promotion of Science (Grants-in-Aid for Scientific Research (C) 24530201, 25380238, and 26380316), and Chuo University (Joint Research Grant 0981). The usual disclaimer applies.

\section{Endnotes}

1. The $n$ by $n$ matrix $\mathbf{B}$ is invertible if $\operatorname{det} \mathbf{B}=(1-\gamma)^{n-1}(1+$ $(n-1) \gamma) \neq 0$. It is clearly invertible when $\gamma>0$. In the case of $\gamma<0$, the inequality constraint $1+(n-1) \gamma>0$ will be assumed in Assumption 10 and it will guarantee the invertibility of $\mathbf{B}$.
2. If the Jacobian of the price function satisfies the diagonally dominant condition (i.e., $\left|d p_{k}\right| d q_{k} \mid>$ $\left.\sum_{i \neq k}\left|d p_{k} / d q_{i}\right|\right)$, then we have

$$
n<1+\frac{1}{\gamma}
$$

under which $\left|\lambda_{n}^{C}\right|<1$. However, we proceed our analysis without this strong assumption.

3. Note that inequality (52) is always fulfilled for $n=2$.

4. $(2+(n-3) \gamma)(1+(n-1) \gamma)-(1+(n-2) \gamma) n \gamma=(1-\gamma)(2+$ $(2 n-3) \gamma)>0$ for $n \geq 1$ and $\gamma<1$.

5. Okuguchi [16] has already shown the same result with a more general demand function.

6. If the Jacobian of the demand function is diagonally dominant (i.e., $\left|d q_{k} / d p_{k}\right|>\sum_{i \neq k}\left|d q_{i} / d p_{k}\right|$ ), then we have

$$
n<\frac{3}{2}-\frac{1}{2 \gamma}
$$

which is stronger than the price-stability condition and thus leads to the stability of Bertrand price. However we do not assume this strong condition in the paper.

\section{References}

[1] R. D. Theocharis, "On the stability of the Cournot solution on the oligopoly problem," Review of Economic Studies, vol. 27, pp. $133-134,1960$.

[2] M. McManus and R. Quandt, "Equilibrium numbers and size in Cournot oligopoly," Yorkshire Bulletin of Social Science and Economic Research, vol. 16, pp. 68-75, 1964.

[3] F. Hahn, "The stability of the Cournot oligopoly solution," Review of Economic Studies, vol. 29, pp. 329-331, 1962.

[4] F. Fisher, "The stability of the Cournot solution: the effects of speeds of adjustment and increasing marginal costs," Review of Economic Studies, vol. 28, pp. 125-135, 1961.

[5] J. Hadar, "Stability of oligopoly with product differentiation," Review of Economic Studies, vol. 33, pp. 57-60, 1966.

[6] W. Krelle, Preistheorie I, J.C.B. Mohr (Paul Siebeck), Tubingen, Germany, 2nd edition, 1976.

[7] K. Okuguchi, Expectations and Stability in Oligopoly Models, Springer, Berlin, Germany, 1976.

[8] K. S. Sridhar Moorthy, "Cournot competition in a differentiated oligopoly," Journal of Economic Theory, vol. 36, no. 1, pp. 86-109, 1985.

[9] J. W. Friedman, Game Theory with Applications to Economics, Oxford University Press, New York, NY, USA, 1986.

[10] R. Selten, Preispolitik der Mehrproduktenunternehmung in der Statischen Theorie, Springer, New York, NY, USA, 1970.

[11] W. Eichhorn, "Zur Statchen Theorie des MehrproductionOligopols," Operations Research Verfahren, vol. 10, pp. 15-33, 1971.

[12] W. Eichhorn, "Zur Dynamischen Theorie des MehrproductenOligopols," Jahbucher fur Nationalokonomie und Statistik, vol. 186, pp. 498-515, 1971. 
[13] F. Szidarovsky, S. Rassenti, and J. Yen, "The stability of the Cournot solution under adaptive expectation: a further generalization," International Review of Economics and Finance, vol. 3, no. 2, pp. 173-181, 1994.

[14] K. Okuguchi and F. Szidarovszky, The Theory of Oligopoly with Multi-Product Firms, vol. 342 of Lecture Notes in Economics and Mathematical Systems, Springer, Berlin, Germany, 1990.

[15] P. K. Goldberg, "Product differentiation and oligopoly in international markets: the case of the U.S. automobile industry," Econometrica, vol. 63, no. 4, pp. 891-951, 1995.

[16] K. Okuguchi, "Equilibrium prices in the Bertrand and Cournot oligopolies," Journal of Economic Theory, vol. 42, no. 1, pp. 128139, 1987.

[17] X. Vives, "On the efficiency of Bertrand and Cournot equilibria with product differentiation," Journal of Economic Theory, vol. 36, no. 1, pp. 166-175, 1985.

[18] L. Cheng, "Comparing Bertrand and Cournot equilibrium: a geometric approach," The RAND Journal of Economics, vol. 16, no. 1, pp. 146-152, 1985.

[19] J. S. Cánovas, T. Puu, and M. Ruíz, “The Cournot-Theocharis problem reconsidered," Chaos, Solitons \& Fractals, vol. 37, no. 4, pp. 1025-1039, 2008.

[20] J. S. Cánovas, "Reducing competitors in a Cournot-Theocharis oligopoly model," Journal of Difference Equations and Applications, vol. 15, no. 2, pp. 153-165, 2009.

[21] G. I. Bischi, C. Chiarella, M. Kopel, and F. Szidarovszky, Nonlinear Oligopolies: Stability and Bifurcations, Springer, Berlin, Germany, 2010. 

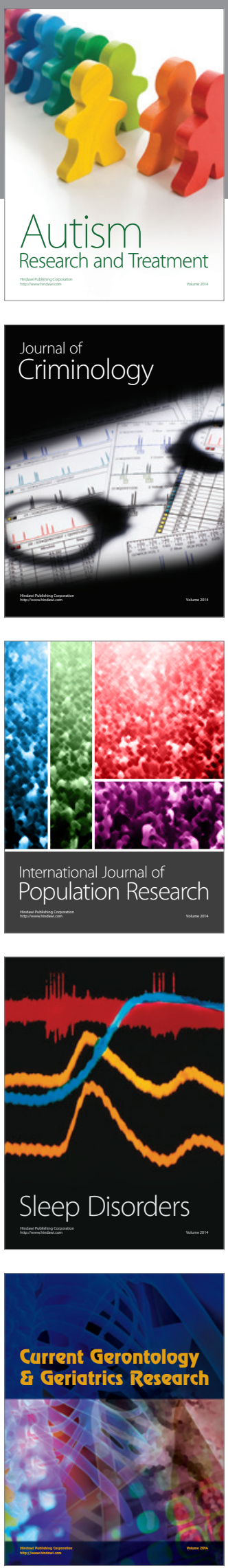
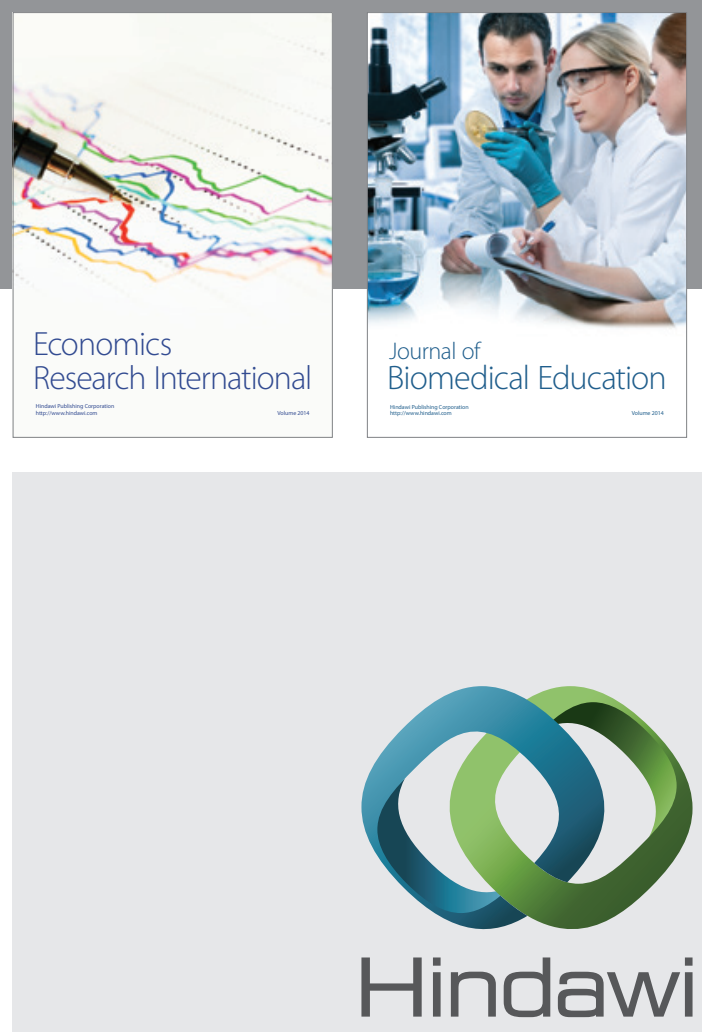

Submit your manuscripts at

http://www.hindawi.com
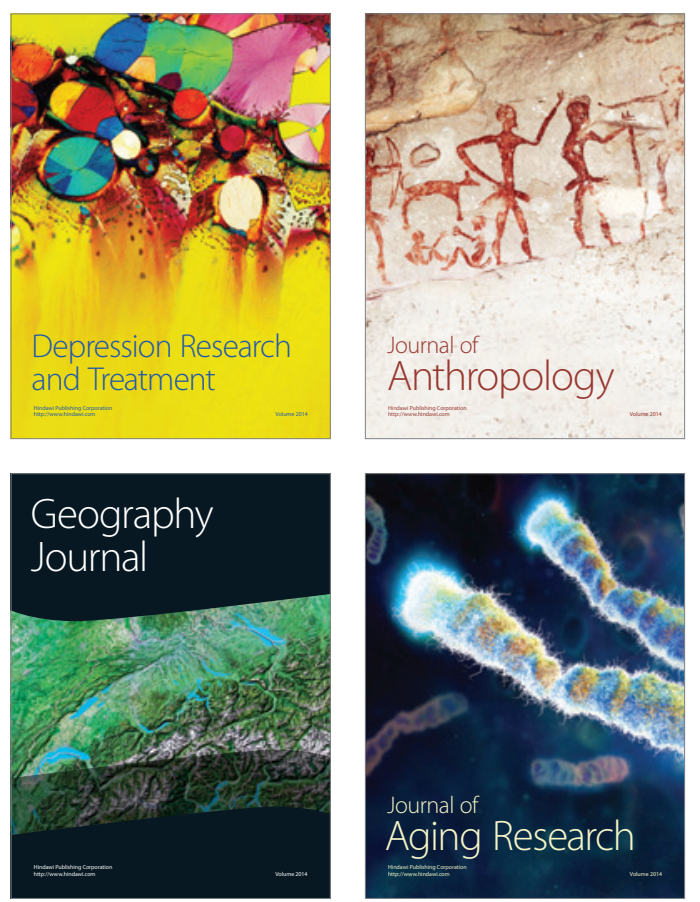
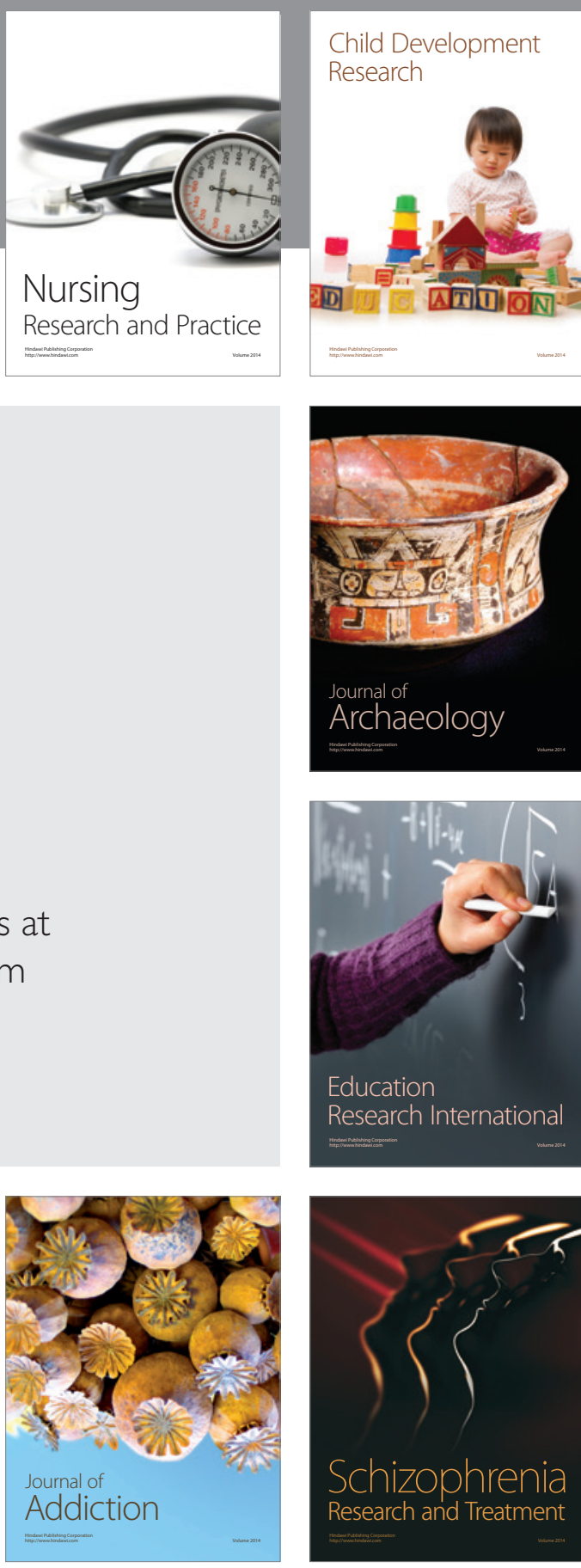

(D)
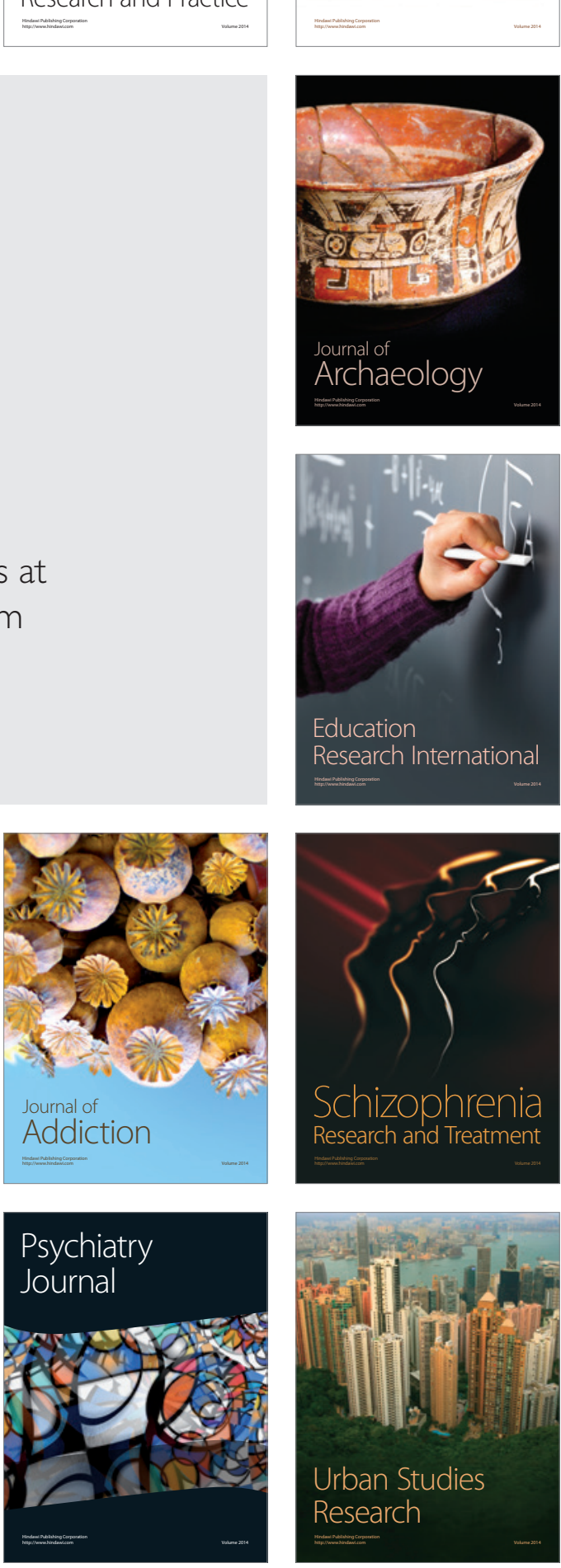